Thorax (1964), 19, 406.

\title{
Variation of drug action on airway obstruction in man
}

\author{
R. E. C. ALTOUNYAN \\ From the Medical Department, Fisons Pharmaceuticals Ltd., Benger Laboratories Division, Holmes \\ Chapel, Cheshire
}

Rapid and sustained relief of airway obstruction is a therapeutic aim which is still only rarely achieved in chronic bronchitis or asthma. Although the topical administration of adrenergic smooth muscle relaxants usually affords a rapid symptomatic improvement in these patients, the duration of relief is limited, and the efficacy of this treatment diminishes as breathlessness increases. There is clearly a need for more effective and longer lasting drugs. This communication is primarily concerned with the variations that were observed in the response to aerosols of isoprenaline sulphate and atropine methonitrate after their administration to patients at different phases of airway obstruction. The protective action of some drugs against artificially induced airway obstruction was also investigated.

\section{METHODS AND MATERIALS}

ESTIMATION OF AIRWAY RESISTANCE Serial F.E.V., and vital capacity values were determined by the author using the same direct writing spirometer throughout. A minimum of three F.E.V.1 recordings were determined for each test before estimating the slow vital capacity, after which a further one to three F.E.V.1 estimations were repeated. The results were plotted to indicate the order of each attempt. When the values progressively diminished, as usually occurred during acute phases of airway obstruction, the F.E.V.1 was calculated as the average of the last three attempts. In the majority of cases, consecutive F.E.V.1 values were similar or increased during the first few attempts ; in these circumstances the average of the three highest values was calculated as the F.E.V.1.

DRUG ADMINISTRATION Drug administration was carried out under strict supervision; aerosols were administered in rooms set aside for this purpose, and only one type of drug was administered in each room. Patients received no isoprenaline for three hours, and no atropine for 12 hours, before tests. All aerosol drugs were administered from a Wright nebulizer operating on compressed air, and a different set of apparatus was used for each type of drug. Aqueous solutions usually contained $5 \%$ propylene glycol$(0.2 \%$ sodium metabisulphite was also included in $\circlearrowleft$ the stock solutions containing isoprenaline).

AEROSOL DOSE The weight loss from the nebulizer and tubing, operating at 6 and $81 . / \mathrm{min}$. air flow, was $\frac{\mathbb{}}{-}$ approximately 150 and $220 \mathrm{mg}$. $/ \mathrm{min}$. respectively. The $\vec{\varphi}$ maximum calculated dose (referred to below) is an approximate estimate of the dose of drug delivered ${ }^{+}$ to the face mask during the inspiratory period. The $\square$ calculation was based on the total weight loss, and losses due to evaporation were not taken into account.

MINIMUM EFFECTIVE DOSE OF ISOPRENALINE SULPHATE 응 AEROSOL Test doses of isoprenaline were admini- $\varrho$ stered during five slow maximal inhalations from a $\overrightarrow{\hat{O}}$ Wright nebulizer operating at $61 . / \mathrm{min}$. air flow (total 3 inspiratory time approximately 15 seconds). In a given $\vec{P}$ subject, the dose inhaled was altered by varying the concentration of the isoprenaline solution between the ranges of 0.05 and $1.0 \%$. The F.E.V.1 was deter- 0 mined three to five minutes after the test aerosol, after which an inhalation of $1.0 \%$ isoprenaline was administered for 60 seconds, and the maximum 3 F.E.V.1 increase from the initial control value was assessed three to five minutes later. The F.E.V.1 im- o provement after the test aerosol was calculated as a percentage of the maximal F.E.V.1 increase. In the majority of tests the administration of a maximum calculated dose equivalent to less than $0.1 \mathrm{mg}$. of isoprenaline was sufficient to induce 90 to $100 \%$ of the maximum F.E.V.1 response. Similar tests were $\sigma$ conducted with a pressurized multidose inhaler which $\mathrm{N}$ delivered at each dose aproximately $0.075 \mathrm{mg}$. iso- $\mathrm{N}$ prenaline sulphate as an aerosol. The minimum num- $\omega$ ber of doses required to induce 90 to $100 \%$ of the $\bar{\gamma}$ maximum response was estimated to lie between one 0 and two. For the studies reported below, a larger dose of isoprenaline was administered: either five breaths of a $1.0 \%$ solution of isoprenaline from the Wright $T$ nebulizer (maximum calculated dose $0.37 \mathrm{mg}$.) or three $\overline{0}$ to four doses from a pressurized multidose inhaler $\mathbb{D}$ $(0.2$ to $0.3 \mathrm{mg}$.)

PROCEDURE FOR ASSESSING DRUG ACTION An increased airway resistance was induced by the aerosol admini- $\Omega$ 
stration of three types of spasmogen: histamine, carbachol or antigen in sensitized patients. The maximum percentage fall in the F.E.V.1 which followed the selected dose of spasmogen aerosol was calculated (control fall). Subsequently, the prophylactic dose of the drug under investigation was followed at varying time intervals by the standard spasmogen challenge, and the maximum percentage fall in the F.E.V.1 was determined (test fall). Drug effect was calculated from the formula

$$
\% \text { protection }=\frac{\text { control fall }- \text { test fall }}{\text { control fall }} \times 100
$$

Control experiments established that histamine challenge sufficient to induce a 20 to $40 \%$ fall in the F.E.V.1 could be repeated at least twice at hourly intervals without any significant alteration in the magnitude of the response. Carbachol has a slightly longer action, but repeatable results followed challenges at 90-minute intervals. A single antigen challenge led to a variable temporary desensitization, but antigen administered at a minimum of three-day intervals was satisfactory. In order to observe the maximum effect, serial F.E.V.1 values were estimated at two, five, and 15 minutes after the start of histamine or carbachol challenge, and at five-minute intervals, for a minimum of 30 minutes, after antigen.

CLASSIFICATION OF PATIENTS Ninety-six patients (61 male, 35 female, aged 14 to 73 years, average age 47 years) with spirometric evidence of airway obstruction were investigated as out-patients. The majority were assessed at regular two- to four-week intervals throughout the year; on the average, each patient in this series attended 59 times over a period of 39 months.

The differential diagnosis between chronic bronchitis and intrinsic or extrinsic asthma was difficult to make on the basis of the history and clinical findings alone; moreover, in some patients each condition could have been considered to be present to a variable degree at different periods. In 12 patients (all under 40 years) with seasonal exacerbations, a diagnosis of extrinsic asthma was confirmed by an aerosol provocation test with the appropriate antigen. However, in eight of these 12 subjects a chronic productive cough with frequent infective episodes during the winter months was also present.

In the older age group, it was even more difficult to evaluate the importance of any underlying intrinsic asthma on clinical grounds alone, especially when chronic bronchitis was also evident. A quantitative estimate of the 'allergic' component (intrinsic and/or extrinsic asthma) of the airway obstruction observed in these patients was obtained during corticosteroid therapy. Ninety-three patients received corticosteroid therapy over a total period of 2,720 treatment months. In the majority, prednisone, 20 to $30 \mathrm{mg}$. daily, was administered initially for seven days, after which the dose was progressively reduced over two weeks to a maintenance level of 7.5 to $15 \mathrm{mg}$. daily. Basal F.E.V.1 values were estimated at approximately seven-day intervals during the first three weeks of each period of corticosteroid therapy. In 87 cases the response was assessed on the basis of the F.E.V.1 observed during the initial three to six weeks of corticosteroid therapy in comparison with the pre-treatment values. Patients already receiving a maintenance dose of corticosteroids were observed at varying dose regimes in an attempt to establish a dose response relationship. The 96 patients in this study have been divided into two groups as follows.

Group $A$ This group consisted of patients responsive to corticosteroid therapy or suffering from proven extrinsic asthma (60 patients, 32 males, aged 14 to 65 , average age 44 years; 28 females, aged 22 to 73 , average age 44 years).

The maximum basal F.E.V.1 increase following corticosteroid therapy was greater than $50 \%$ in 36 patients and 10 to $50 \%$ in 16 patients. In six cases, corticosteroid therapy could not be withheld altogether owing to the severity of their condition, but evidence of a response to corticosteroids was established at varying dosage. Two patients with proven extrinsic asthma were never sufficiently distressed to justify a trial of corticosteroid therapy.

Group $B$ These patients, 36 in all, were not responsive to corticosteroids (29 males, aged 31 to 67 , average age 50 years; 7 females, aged 39 to 60 , average age 47 years).

Twenty-eight patients showed no evidence of any improvement after corticosteroid therapy. In seven cases, the improvement was not greater than $10 \%$. One male patient, who was not treated with corticosteroids because of diabetes, has been included in this group as he had no history of allergy and presented with classical chronic bronchitis.

Routine MANAGEMENT Purulent sputum was regarded as evidence of infection. Patients were instructed to examine their sputum daily, and to commence tetracycline therapy on their own initiative $(1.5$ to $2.0 \mathrm{~g}$. daily for three days followed by 1.0 to $1.5 \mathrm{~g}$. for four days) and report for sputum culture if improvement did not occur within three days. In some cases, frequent relapses of infection necessitated maintenance therapy $(0.75$ to $1.5 \mathrm{~g}$. daily), particularly during the winter months. Patients who responded to corticosteroids were maintained on the lowest dose consistent with reasonable comfort (5 to $15 \mathrm{mg}$. prednisone daily). Long-term therapy with oral ephedrine, antihistamines, aminophylline suppositories, and isoprenaline inhalations was continued when a worth-while improvement was claimed by the patient. Chronically dyspnoeic patients were maintained on dichlorphenamide, 25 to $200 \mathrm{mg}$. daily, when this treatment effected a significant improvement in exercise tolerance, vital capacity, and $\mathrm{PCO}_{2}$ values.

\section{RESULTS}

ISOPRENALINE SULPHATE

Protective action against spasmogen challenge Figs. 1, 2, and 3 summarize some of the results 


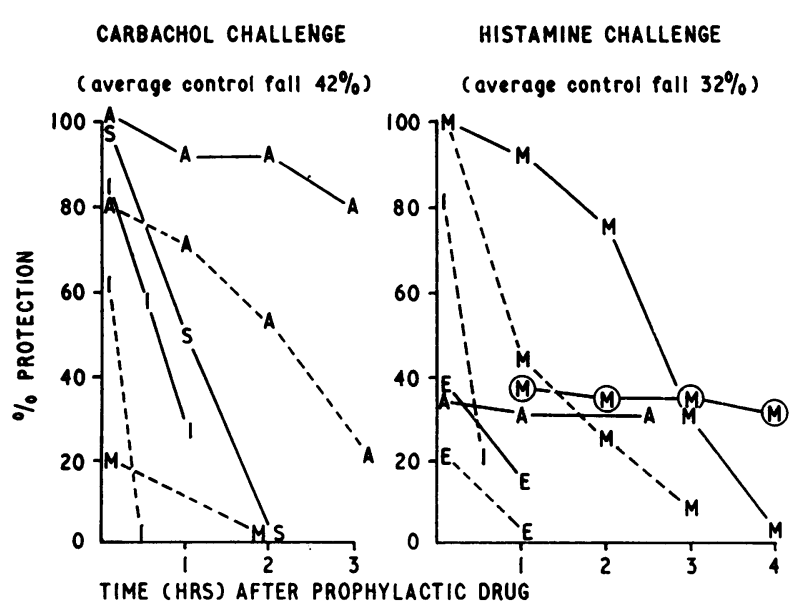

FIG. 1

FIG. 2

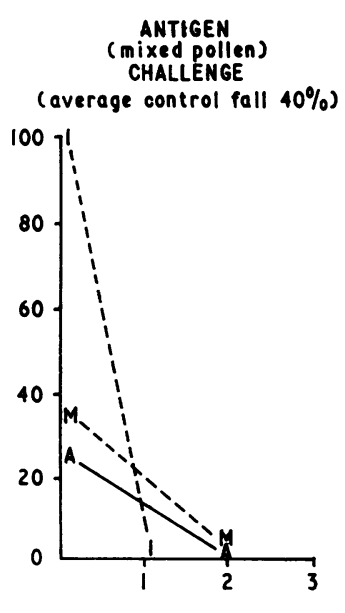

FIG. 3

FIGS. 1, 2, and 3. Protection afforded by prophylactic administration of drugs (as indicated) against spasmogen challenge.

\begin{tabular}{|c|c|c|c|}
\hline \multirow{3}{*}{$\begin{array}{l}\mathbf{A} \longrightarrow \mathbf{A} \\
\mathbf{A} \ldots \mathbf{A}\end{array}$} & & & max. calculated dose (mg.) \\
\hline & Atropine metho & onitrate $0.2 \%$ & 0.05 \\
\hline & & $0.1 \%$ & 0.025 \\
\hline $\mathbf{S}-\mathbf{S}$ & Atropine sulph & $0.2 \%$ & 0.05 \\
\hline I-I I & Isoprenaline & $1.0 \%$ & $3 \cdot 0$ \\
\hline I - . . - I I & ,, & $0 \cdot 1 \%$ & $0 \cdot 3$ \\
\hline
\end{tabular}

of a series of tests with various bronchodilator compounds which were conducted on the author. Essentially similar results were obtained in a group of volunteer patients although all tests could not be completed on the same individual for accurate comparison. In Figs. 1, 2, and 3, isoprenaline administration at a clinical dose is shown to be effective against all three spasmogens when administered five to 10 minutes before the challenge. The duration of protection was, however, brief and rarely exceeded 60 minutes in any experiment. The administration of a massive dose of isoprenaline, equivalent to 10 times the clinical dose, afforded only a marginal increase in the magnitude and duration of effect, even though serious side effects were induced.

Duration of clinical response After a rest period of at least 20 minutes, the basal F.E.V.. was determined ; isoprenaline was then administered, and the F.E.V. ${ }_{1}$ was estimated approximately five minutes later and thereafter at intervals of 20 to 60 minutes. The duration of effect was interpolated from these values. The time taken for the F.E.V..$_{1}$ to decline to a point half way between the basal F.E.V..$_{1}$ and the maximum F.E.V..$_{1}$ value was designated the duration of $50 \%$ effect.
Whenever possible, the duration of the iso- $\overrightarrow{\overrightarrow{0}}$ prenaline response was estimated in the same patient at widely different phases of disease. The results from groups A and B patients are shown separately in Figs. 4 and 5 respectively. When the basal F.E.V..$_{1}$ did not vary more than $20 \%$ the results are represented by a single point for each $\stackrel{\times}{-}$ patient. When the basal F.E.V..$_{1}$ varied more than 3 $20 \%$ on different occasions in the same subject, the results representing the widest variation are $\frac{3}{3}$ shown ; these are identified as points connected by a continuous line. The results clearly indicate that the duration of relief afforded by isoprenaline was $\frac{D}{O}$ significantly reduced during phases of relatively increased airway obstruction. The duration of $N$ $50 \%$ effect rarely exceeded 60 minutes in patients $\mathrm{N}$ of either group, but during the more acute phases it was usually 30 minutes or less. Spasmogen $\omega$ challenge experiments were conducted in a group of selected patients to determine whether the

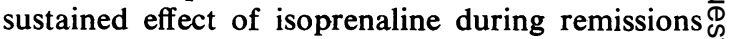
could be attributed to slower inactivation of the ${ }^{-}$ drug. The results from one subject are shown in $\frac{0}{0}$ Figs. 1, 2, and 3, in which little or no protection $\overrightarrow{\mathbb{D}}$

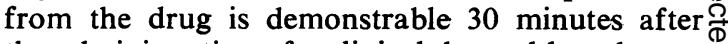
the administration of a clinical dose, although over $\cong$ $50 \%$ of the F.E.V. ${ }_{1}$ increase induced by iso 
GROUP A

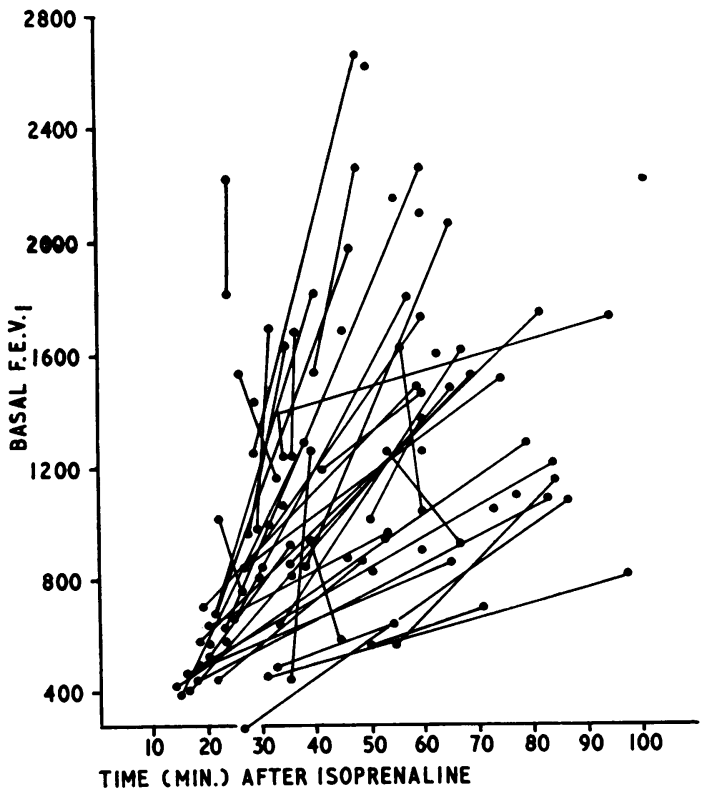

FIG. 4

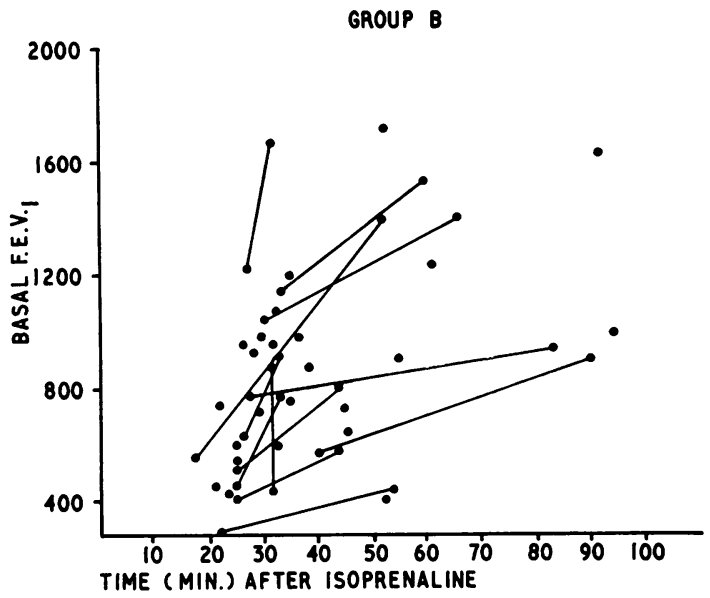

FIG. 5

FIGS. 4 and 5. Duration of response to isoprenaline inhalations of group $A$ and $B$ patients respectively, in relation to initial airway obstruction.

prenaline administration was maintained for up to 95 minutes in this subject. The duration of a significant degree of isoprenaline-induced protection against spasmogens did not exceed 60 minutes in any of the test subjects.

Cholinergic blocking agents Seventeen tropane derivatives, three synthetic tertiary and quaternary amine anticholinergic compounds, an antimuscarine (benzhexol), a ganglion-blocking agent (pentolinium) and several antihistamines (e.g., mepyramine) were investigated for bronchodilator activity. Relative potency was assessed from the magnitude and duration of the F.E.V. increase, observed over a four-hour period following aerosol administration of the compounds at comparable concentrations, which varied from 0.2 to $1.0 \%$. The tropane class of compounds was found to be the most active. The antihistamines, synthetic anticholinergic, and antimuscarine compounds all showed some transient activity, whereas pentolinium had no detectable action under these experimental conditions. Analogous atropine and hyoscine derivatives were approximately equi-active, homatropine less active, and tropine derivatives almost inactive. Increasing the $\mathrm{N}$-alkyl chain from methyl to ethyl in the atropine series resulted in a significant loss of activity. Similarly, N-butyl hyoscine was less active than $\mathrm{N}$-methyl hyoscine. The duration of effect was significantly prolonged by quaternization (Figs. 1 and 6). The increased duration of topically applied

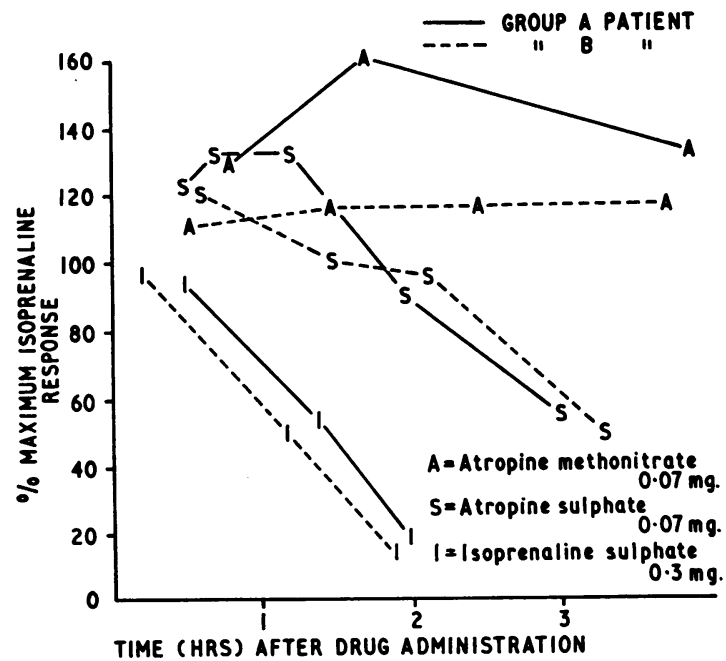

FIG. 6. Comparison of magnitude and duration of changes in F.E.V.1 after aerosol administration of isoprenaline, atropine sulphate, and atropine methonitrate in two patients.

quaternary compounds was also demonstrated by a derivative of mepyramine: mepyramine methyl bromide (quaternary salt) is less active as an antihistamine on a weight basis than mepyramine maleate (tertiary salt), but it has a more prolonged effect when compared with the tertiary salt (Fig. 2).

It was concluded that the potent and sustained 
activity of atropine methonitrate merited further investigation.

\section{ATROPINE METHONITRATE}

Protective action against spasmogen challenge The protection afforded by aerosol atropine methonitrate against carbachol, histamine, and antigen challenge in the same subject is shown in Figs. 1, 2, and 3 respectively.

Atropine is clearly more specific in its action as compared with isoprenaline, and although some protection against both histamine and antigen challenge was demonstrated it is of a low order. Clinical response to atropine methonitrate aerosol The approximate minimum dose of atropine methonitrate that could induce a maximum F.E.V. ${ }_{1}$ increase was investigated by the administration of aerosols containing varying concentrations of the drug. Cartridges with a known quantity of atropine as a finely divided powder were also prepared, so that a predetermined quantity of drug could be dispersed into the inspired air stream.

In the majority of tests, it was found that a maximum calculated dose of between 0.005 and $0.05 \mathrm{mg}$. of atropine was sufficient to induce a maximum effect.

In some cases the maximum response was not apparent for 30 to 60 minutes after atropine administration. Larger doses, or the administration of tertiary atropine salts, e.g., atropine sulphate, did not significantly affect the time required for the maximum response to develop. The maximum increase in the F.E.V..$_{1}$ was assessed in 94 patients after five inspirations of a $\mathbf{0 \cdot 2} \%$ aqueous aerosol of atropine methonitrate (maximum calculated dose, $0.07 \mathrm{mg}$.). The maximum atropine response was compared with the maximum response after a clinical dose of isoprenaline alone. In the majority of tests, each drug was assessed from identical pre-treatment F.E.V.$_{1}$ values, but where this was not possible, the average of two isoprenaline responses within $\pm 5 \%$ of the basal F.E.V..$_{1}$ value recorded for the atropine test was accepted. When isoprenaline was administered first, both tests could be completed on the same day, and the results obtained in this manner agreed closely with those obtained on different days from similar basal F.E.V.. values.

Figures 7 and 8 show the results of 60 group $A$ and 34 group B patients respectively. Different symbols indicate the relative degree of basal airway obstruction prevailing in each patient at the time of the test; this assessment was based on the previous records which extended for periods up to five years.

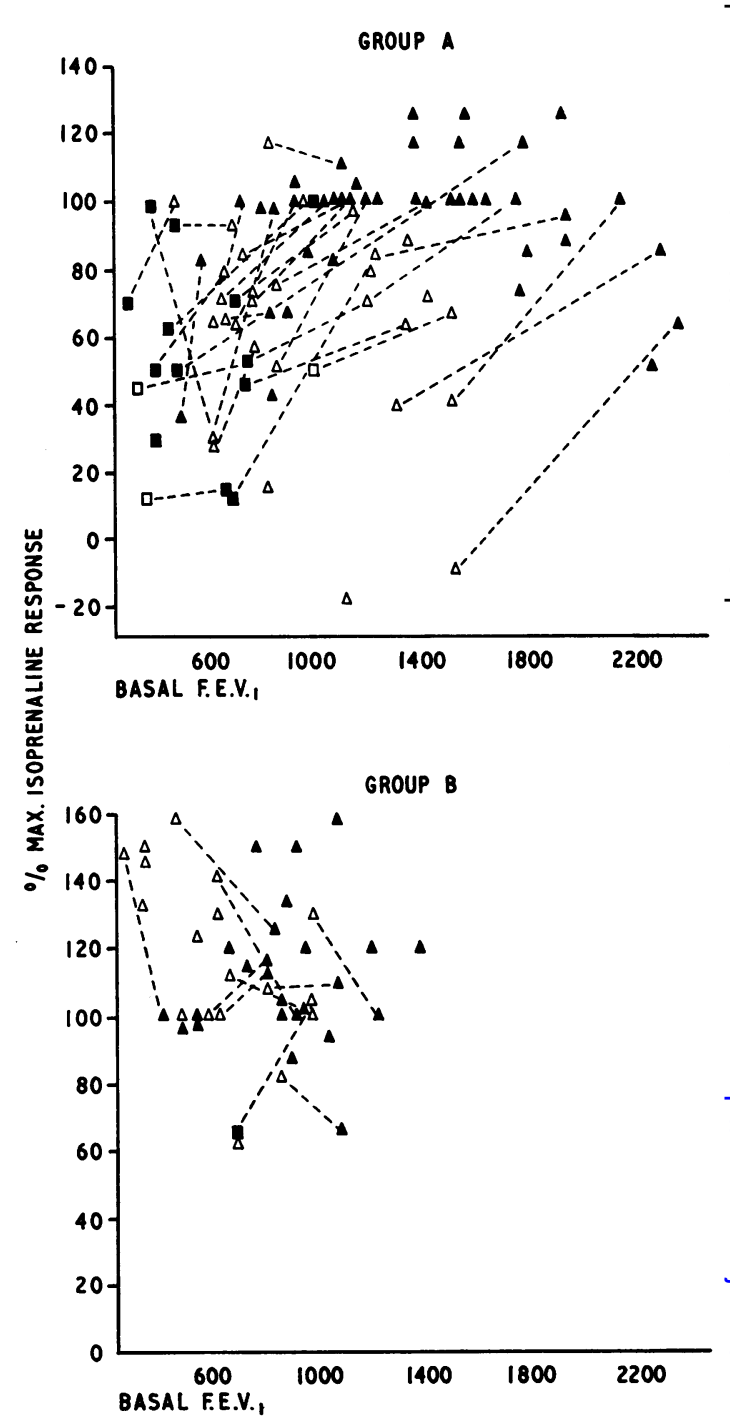

FIGS. 7 and 8. Magnitude of response in group $A$ and $B \frac{D}{2}$ patients respectively, to inhalations of atropine expressed as a percentage of the maximum response to isoprenaline $\mathrm{N}$ from similar initial F.E.V. $\cdot_{1}$ values. The phase of airway $N$ obstruction in each patient at the time of test is indicated $\mathrm{N}$ by the following symbols. A 75 to $100 \%$ maximum $\mathrm{\omega}$ recorded F.E.V.; $\triangle 50$ to $74 \%$; $\square 25$ to $49 \%$; 口 below $25 \%$. Interrupted lines connect results from the same patient tested at significantly different phases of disease.

The efficacy of atropine methonitrate clearly differs in the two groups under study.

Group A patients (Fig. 7) showed a variable response. The response to atropine was as great as, or exceeded, that to isoprenaline when these patients were in relative remission. The same 
patients tested during periods of increased airway resistance showed a significant and progressive decline of the atropine response compared with the isoprenaline response (assessed from similar basal conditions). Two patients in this group, as well as two similar cases not included in this series, showed a negative response to atropine. The inhalation of an aerosol of distilled water also effected some increase in the airway resistance in these patients.

Group B patients (Fig. 8) showed a more uniform response. In the majority of these subjects, atropine induced an effect equal to or greater than the corresponding isoprenaline response. There did not appear to be any correlation between the prevailing degree of airway obstruction and the relative efficacy of atropine in group B patients.

Duration of response to aerosols of atropine methonitrate combined with isoprenaline sulphate (a) Administered from the Wright nebulizer Atropine methonitrate $0.2 \%$ and isoprenaline $1.0 \%$ were administered at a standard dose (maximum calculated dose of atropine methonitrate, $0.07 \mathrm{mg}$; isoprenaline sulphate, $0.37 \mathrm{mg}$.). The initial increase in the F.E.V. approximately five minutes after the aerosol was recorded, and the estimations were repeated at 30- to 60-minute intervals for as long as practicable. The results from 50 group $A$ and 31 group B patients are plotted in Figs. 9 and 10 respectively. The residual effects (expressed as percentages of the maximum increases in the F.E.V..$_{1}$ recorded during the test day) are plotted as ordinates; the period of observation after drug administration as abscissae. The symbols (see legend to Figs. 7 and 8 ) signify the relative degree of airway obstruction prevailing in each patient at the time of test; interrupted lines connect results from the same patient when records were obtained at significantly different phases of disease.

Group A patients (responsive to corticosteroids, Fig. 9) showed considerable variation in their responses. The duration of effect was apparently correlated with the relative degree of airway obstruction prevailing in each patient at the time of test. The majority showed a sustained effect, which persisted for several hours when they were well, but the duration of effect was progressively reduced as the relative degree of basal airway obstruction increased. In contrast, in the majority of group B patients (not responsive to corticosteroids, Fig. 10) a sustained response was maintained even during phases of relatively increased airway obstruction.

(b) Administered from a pressurized multi-dose

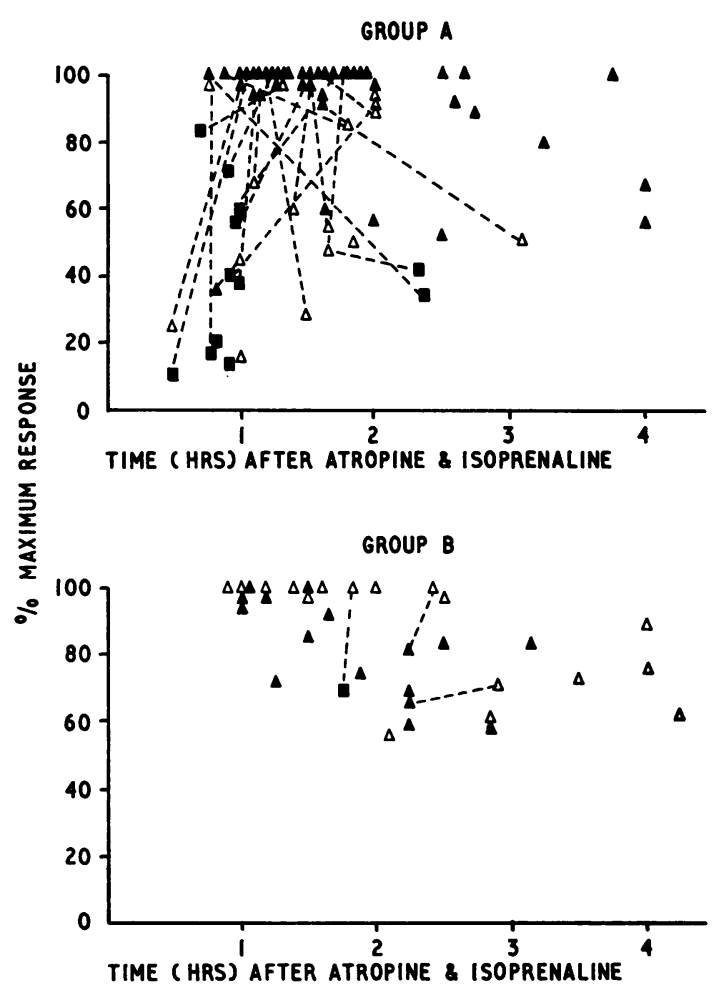

FIGS. 9 and 10. Rate of decline of the response in group $A$ and $B$ patients respectively to inhalations of isoprenaline and atropine.

inhaler The inhaler employed delivered an aerosol containing $0.04 \mathrm{mg}$. atropine methonitrate and $0.1 \mathrm{mg}$. isoprenaline sulphate at each dose. A valve mechanism was incorporated in the mouthpiece to facilitate the co-ordination of drug release with the commencement of inspiration. In a series of tests on 18 patients (10 group $B$ and eight group A, during a phase of relative remission) the efficacy of this inhaler was compared with that of a similar appliance which delivered isoprenaline alone.

Each type of therapy was administered on a different day, in a random order, over a four-week period. On test days, patients were asked to use isoprenaline alone when relief was necessary in the early morning, and they were requested not to inhale this drug after 8 a.m. Basal F.E.V. values were determined at 11 a.m. and were repeated approximately one hour later. When there was more than $10 \%$ difference between the two F.E.V. values, the test was abandoned on that day. When the difference was less than $10 \%$, the basal F.E.V.: value was calculated from the 


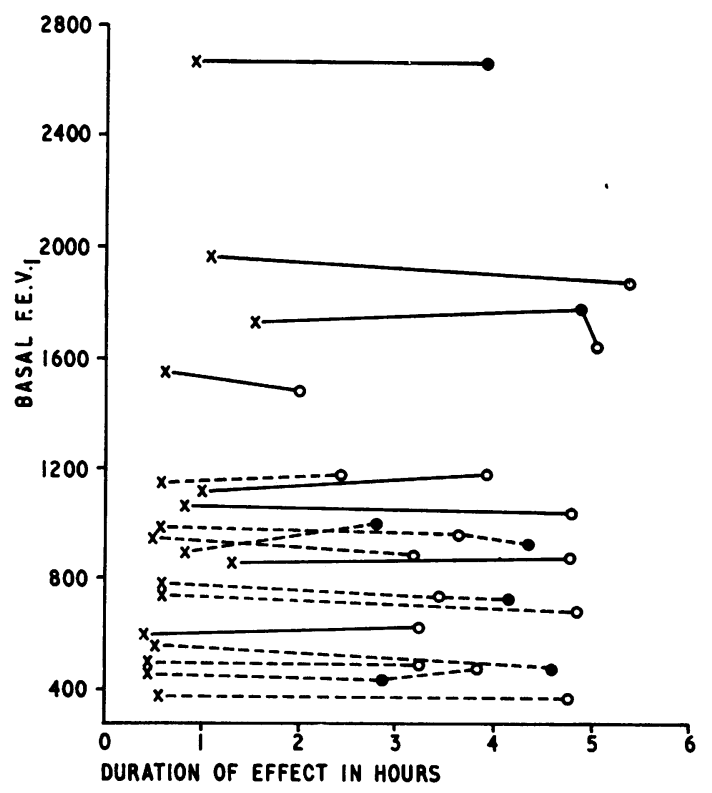

FIG. 11. Duration of response in eight group $A$ and 10 group $B$ patients after inhalations of isoprenaline alone compared with isoprenaline and atropine, administered from a pressurized multi-dose inhaler. $\times$ Isoprenaline alone (max. calculated dose $0.2 \mathrm{mg}$.); C Isoprenaline and atropine -one dose (max. calculated dose 0.1 and $0.04 \mathrm{mg}$. respectively); Isoprenaline and atropine-two doses. The results from each group $A$ patient are joined by a continuous line, those of group $B$ patients by interrupted lines.

second attempt. The test inhalation was then administered by an assistant, and the F.E.V..$_{1}$ was reassessed after intervals of five minutes, 30 minutes, and thereafter approximately hourly for six hours or until the F.E.V..$_{1}$ had reverted to pre-treatment values.

A clinically significant residual effect was considered to be equivalent to $50 \%$ of the maximum increase in the F.E.V..$_{1}$ after the administration of isoprenaline alone. The duration of a response which never fell below this value at any period was determined by interpolating the serial F.E.V., values obtained after each type of therapy. The results in each patient are shown in Fig. 11.

The administration of the combined drugs clearly afforded a considerably longer period of relief in all the patients tested. There was no evidence that this effect could be significantly prolonged by increasing the dose of the combined drugs. It should be emphasized that atropine offers no advantage during acute phases of airway obstruction in corticosteroid-responsive ('allergic') patients. This type of patient often inhales $\frac{}{0}$ isoprenaline every few minutes in an attempt to

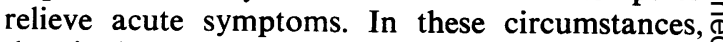
the indiscriminate use of aerosols containing atropine could lead to a serious over-dosage.

PAPAVERINE $\mathrm{HCl}$ Papaverine $\mathrm{HCl}$ is classed as a $\overrightarrow{\vec{H}}$ non-specific smooth muscle relaxant on the basis of pharmacological tests. It is widely used clinic- $\overrightarrow{+}$ ally and included in the National Formulary in $\times$ Nebula Isoprenalinae Sulphatis Composita B.P.C. $\overrightarrow{0}$ as a $2.5 \%$ solution. Aerosols of $2.5 \%$ papaverine (maximum calculated dose, $1.2 \mathrm{mg}$.) were admini- $\vec{\circ}$ stered to 12 patients. An increase in airway resistance occurred in six patients; the remainder showed no significant improvement.

EPHEDRINE $\mathrm{HCl}$ Insomnia, palpitation, and $\stackrel{\vec{\Phi}}{\vec{D}}$ urinary retention in elderly male patients pre- $\frac{3}{\sigma}$ cluded effective oral therapy in the majority of $\stackrel{\mathbb{\Phi}}{-}$ cases. Aerosol administration was ineffective at $\vec{c}$ low doses. The low order of protection afforded $\$$ by higher doses administered by aerosol against histamine challenge is shown in Fig. 2. The combination of ephedrine with isoprenaline by aerosol afforded no significant increase in the magnitude or duration of the isoprenaline response.

MISCELLANEOUS COMPOUNDS In the majority of tests, oral or intravenous aminophylline, intramuscular pethidene or mepyramine maleate, and sublingual glyceryl trinitrate administered at clinical doses were all less effective than isoprenaline and/or atropine.

HISTAMINE AEROSOL CHALlenge A consistent hypersensitivity to histamine aerosol (maximum calculated dose, $0.017 \mathrm{mg}$. histamine base) was demonstrated in nearly all group A and B patients tested repeatedly at intervals throughout the year. Patients with seasonal asthma were the exception, as they showed a marked increase in sensitivity ơ coincident with the onset of the asthma season. In two of these cases the increase in the sensiti- $N$ vity to histamine was shown to parallel a corre- $\omega$ sponding increase in sensitivity to specific antigen ; both forms of hypersensitivity persisted during $\stackrel{\circ}{\subset}$ the asthma season in spite of corticosteroid $\mathbb{D}$ therapy which was adequate to restore the basal : F.E.V..$_{1}$ to within the normal range. In contrast, $\frac{0}{0}$ in patients with proven grass-pollen sensitivity, $\stackrel{\vec{D}}{\mathbb{D}}$ who showed evidence of persistent airway obstruc-

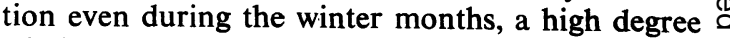
of histamine sensitivity was demonstrated through- 
out the year. Tests with aerosols of carbachol, citric acid, propylene glycol, and other irritants confirm the clinical impression that chronic air flow obstruction is associated with a persistent hypersensitivity to a wide variety of irritants.

\section{DISCUSSION}

The short action of isoprenaline reported above confirms the observations of Lowell, Curry, and Schiller (1949), and of Bresnick, Beakey, Levinson, and Segal (1949), who conducted spasmogen challenge experiments after the administration of oral, parenteral, and aerosol isoprenaline.

Rapid metabolic inactivation of isoprenaline can be observed in sputum (expectorated a few minutes after isoprenaline inhalations) in which the pink-brown adrenochrome derivates are sometimes mistaken for blood. This rapid degradation together with diffusion probably accounts for the absence of any significant prolongation of action after the administration of large doses of isoprenaline. By contrast, mepyramine, which is not actively metabolized in the lung, showed a significantly longer duration of action when the dose was increased (Fig. 2). Attempts to block the enzymatic degradation of isoprenaline by the simultaneous administration of ortho-methyltransferase inhibitors have been unsuccessful (unpublished results), but it is possible that this approach can lead to the development of longeracting adrenaline derivatives.

The prolongation of therapeutic action beyond the period when its presence can be demonstrated by spasmogen challenge tests implies that the factors responsible for airway obstruction are acting slowly. The duration of this 'free wheel' period, during which a reduced airway resistance is apparently maintained in the absence of drug, is an indication of the activity of the noxious processes causing airway obstruction; when the duration of $50 \%$ effect is less than 45 minutes, considerable improvement may be anticipated; a similar decrease occurring at 90 minutes or later indicates that the patient is in a phase of relative remission.

Atropine, or its analogues, has been used to relieve respiratory distress for over 150 years (Finnegan, 1950), but conflicting reports of its therapeutic value continue to appear (Palmer, 1962 ; Chamberlain, Muir, and Kennedy, 1962). Some of the apparent discrepancies in the literature can be explained on the basis of the results presented above. Hume and Jones (1961) administered atropine as a $4.0 \%$ aqueous aerosol but
F.E.V. ${ }_{1}$ changes were estimated for only 15 minutes after drug administration, so that the maximum effect of atropine may not have been observed in some cases. The side effects which these workers reported were not observed in the present study in which much lower doses were used.

Herxheimer (1959), who administered atropine sulphate as a smoke as well as as a wet aerosol, reported an effective duration of less than three hours. His results with atropine sulphate were confirmed in this study (Fig. 6) ; the quaternary salt, however, was shown to have a longer duration, lasting up to six hours.

It is apparent that a number of factors can influence the outcome of atropine therapy ; failure to recognize these factors has led to conflicting opinions in the past. The selection of patients, the phase of disease, the choice of atropine derivative, the dose and method of administration, and the period of observation can all affect the results.

The mechanism of action of atropine therapy on airway obstruction is of interest. Armitage, Herxheimer, and Rosa (1952) reported that atropine has significant anti-anaphylactic properties when tested by the micro-shock technique on guinea-pigs, but the potent antihistamines were much more effective. The anti-anaphylactic properties of atropine in the guinea-pig can perhaps be attributed entirely to its weak antihistaminic action. In man (Fig. 3), both atropine and potent antihistamines are virtually ineffective against experimentally induced allergic airway obstruction. This suggests that histamine is probably not the major spasmogen released from human lung after anaphylactic challenge (Brocklehurst, 1956). On the other hand, Dautrebande, Lovejoy, and McCredie (1962) have demonstrated that atropine sulphate is as effective as sympathomimetic drugs in preventing the airway changes induced by aluminium powder inhalation in normal and emphysematous patients. Thus the efficacy of atropine depends primarily on the basic aetiology of the airway obstruction. The similar response to atropine of group A patients during a remission and group B patients suggests that the same mechanism underlies the increased airway resistance in these cases. However, during exacerbations of airway obstruction in group A patients, a different mechanism operates, which is resistant to atropine but sensitive to corticosteroid therapy.

The slow onset of the atropine response suggests that its action is not merely due to parasympathetic blockade. A possible explanation of its mode of action is suggested by the work of Vaughan 
Williams (1961) on isolated guinea-pig atrial muscle. He reported that the contractility and electrical behaviour of the muscle was altered in opposite directions by acetylcholine and histamine. Most of these effects, he considers, could be attributed to an increase in membrane permeability to potassium and sodium induced by acetylcholine and histamine respectively. It is possible that the contractile properties of human bronchial muscle are also influenced in opposite directions by histamine and acetylcholine. Thus, although atropine is an effective 'competitive inhibitor' at the acetylcholine receptor site, and correspondingly less effective at the histamine receptors, it may also alter the basic tone of the muscle fibre by opposing the permeability changes induced by acetylcholine. The slow onset of the atropine response, as measured by F.E.V..$_{1}$ increase, in contrast to its immediate effect against carbachol challenge, may be accounted for by the time required to establish a new equilibrium in the muscle fibres after a change in permeability. Absence of effect and, in some cases, an increased airway resistance which follows the administration of atropine to 'allergic' subjects may also be explained on this basis. These subjects liberate excessive quantities of histamine (as shown by eosinophilic sputum), so that under these abnormal conditions the consequences of the permeability changes induced by histamine may be partially held in check by the opposing action of acetylcholine; the administration of atropine could then enhance the action of histamine and lead to increased airway obstruction.

From the clinical standpoint, estimations of the relative efficacy and duration of action of atropine methonitrate, in comparison with isoprenaline, can help to distinguish between 'allergic' and 'nonallergic' airway obstruction. When a patient is already receiving corticosteroids, a good atropine response implies adequate control; a poor response to atropine indicates inadequate corticosteroid control, possibly as a result of other factors such as infection.

Quantitative estimations of airway obstruction induced by specific antigen or non-specific spasmogen challenge can be of assistance in the assessment of therapy. It is evident that curative therapy will reduce the abnormally high level of airway irritability which is observed in all patients with 'chronic bronchitis' or asthma.

\section{SUMMARY OF RESULTS}

The magnitude and duration of effect of isoprenaline, atropine derivatives, and three types of spas- mogen administered as aerosols were estimated in a group of patients at varying phases of increased을 airway resistance. The type of response was shown to depend on the relative degree of obstruction and the aetiology of the condition.

ISOPRENALINE THERAPY Inhalations of isoprenaline induced some rapid improvement in all forms $\vec{\omega}$ of spasmodic airway obstruction. The duration of positive effect, as shown by the presence of ax significant level of protection against spasmogen $\overrightarrow{\vec{\theta}}$ challenge, was limited to approximately 30 . minutes.

The duration of the F.E.V..$_{1}$ increase after iso- $\circ$ prenaline inhalation served as a useful index of the relative degree of airway obstruction whichwas present in a given patient at the time of test. $\mathbb{D}$ No clinically significant prolongation of effect was achieved by increasing the dosage up to the limit 3 of tolerance.

ATROPINE THERAPY Atropine was shown to be a poor antagonist of both antigen and histamine induced airway obstruction.

Compared with isoprenaline, atropine therapy afforded significantly greater relief as well as aō more prolonged effect in patients not susceptible⿳ूँ to corticosteroids. Patients who benefit from $\mathscr{\mathbb { Q }}$ corticosteroids also responded well to atropine $\overrightarrow{\vec{\sigma}}$ during phases of relative remission.

The action of quaternary atropine derivates, e.g., atropine methonitrate, administered topically? was sustained longer than that of the corresponding tertiary salts, e.g., atropine sulphate.

Of the commonly used antispasmodic drugs, isoprenaline and atropine were found to be the most effective.

SPASMOGEN CHALLENGE Histamine and other spasmogen challenge tests have provided precise,, quantitative information on the mechanism of action and duration of effect of drugs acting on the bronchial musculature.

Hypersensitivity to irritants was observed of both 'allergic' and 'non-allergic' airway obstructive disease. Fluctuations of the basic level of suscepti- $\omega$ bility to irritants, induced by therapy or other factors, were assessed quantitatively by determining changes in sensitivity to standard spasmogen challenge.

Measurement of the magnitude of the response $\frac{0}{T}$ to atropine methonitrate compared with isoprena- $\frac{\text { D }}{\mathbb{D}}$ line alone can be used to identify and assess the progress of patients who will respond to cortico- $\varrho$ steroid therapy. 
I wish to thank Dr. W. Robinson for permission to study his patients and for his help in providing facilities for this investigation at Monsall Hospital, Manchester. Dr. J. B. L. Howell read the manuscript ; his valuable criticisms are acknowledged with gratitude. I am also deeply indebted to the patients and to Staff Nurse T. Bardsley for their most willing co-operation.

This work was financed by Benger Laboratories Limited. who also manufactured the pressurized multi-dose inhaler containing isoprenaline and atropine (Registered trade mark: Prenomiser Plus). I am grateful for the assistance of Mr. L. Martin and Dr. G. P. Ellis, of the Research Department, and to the directors of the company for permission to publish.

\section{REFERENCES}

Armitage, P., Herxheimer, H., and Rosa, L. (1952). The protective action of antihistamines in anaphylactic microshock of the guinea-pig. Brit.J. Pharmacol., 7, 625.
Bresnick, E., Beakey, J. F., Levinson, L., and Segal, M. S. (1949). Evaluation of therapeutic substances employed for the relief of bronchospasm V. Adrenergic agents. J. clin. Invest., 28, 1182 .

B1 ocklehurst, W. E. (1956). A slow reacting substance in anaphylaxis "SRS-A." In Ciba Foundation Symposium on Hista mine, $\mathrm{p} 175$. Churchill, London.

Chamberlain, D. A., Muir, D. C. F., and Kennedy, K. P. (1962). Atropine methonitrate and isoprenaline in bronchial asthma. Lancet, 2, 1019.

Dautrebande, L., Lovejoy, F. W, and McCredie, R. M. (1962). Effects of atropine microaerosols on the airway resistance in man. Arch. int. Pharmacodyn., 139, 198.

Finnegan, J. K. (1950). Stramonium cigarettes and powders. Bull. nat. Formulary Comm., 18, 131.

Herxheimer, H. (1959). Atropine cigarettes in asthma and emphysema. Brit. med. J., 2, 167.

Hume, K. M., and Jones, E. Rhys (1961). The response to bronchodilators in intrinsic asthma. Quart. J. Med., 30, 189

Lowell, F. C., Curry, J. J., and Schiller, I. W. (1949). A clinical and experimental study of isuprel in spontaneous and induced asthma. New Engl. J. Med., 240, 45.

Palmer, K. N. V. (1962). Current Therapeutics 178-. Aerosols in chronic bronchitis. Practitioner, 189, 546.

Vaughan Williams, E. M. (1961). The action of quinidine, acetylcholine and anaphylaxis interpreted from simultaneous records of contractions and intracellular potentials in the heart. Sci. Basis Med. Ann. Rev., p. 302. Athlone Press, London. 